\title{
Photometric amplitudes and phases of nonradial oscillation in rotating stars
}

\author{
J. Daszyńska-Daszkiewicz ${ }^{1,2}$, W. A. Dziembowski ${ }^{2,3}$, A. A. Pamyatnykh ${ }^{2,4}$, and M.-J. Goupil ${ }^{5}$ \\ 1 Astronomical Institute of the Wrocław University, ul. Kopernika 11, 51-622 Wrocław, Poland \\ 2 Copernicus Astronomical Center, Bartycka 18, 00-716 Warsaw, Poland \\ 3 Warsaw University Observatory, Al. Ujazdowskie 4, 00-478 Warsaw, Poland \\ ${ }^{4}$ Institute of Astronomy, Russian Academy of Sciences, Pyatnitskaya Str. 48, 109017 Moscow, Russia \\ 5 DASGAL, UMR CNRS 8633, Observatoire de Paris-Meudon, France
}

Received 28 May 2002 / Accepted 11 June 2002

\begin{abstract}
Effects of rotational mode coupling on photometric parameters of stellar oscillations are studied. At moderate rotation rates, a strong coupling between modes of spherical harmonic degree, $\ell$, differing by 2 and of the same azimuthal order, $m$, takes place if the frequencies are close. This is a common situation amongst main sequence pulsators. Numerical results for a sequence of $\beta$ Cephei star models are reported for the two- and three-mode couplings.

One consequence of mode coupling is that modes of higher degree should be considered in photometric mode identification. Modes with nominal degree $\ell>2$ acquire substantial $\ell \leq 2$ components and therefore are more likely to reach detectable amplitudes. Coupled mode positions in the amplitude ratio - phase difference diagrams, based on multicolour photometry, become both aspect- and $m$-dependent. Examples of the mode path in the diagram with varying aspect are given. The diagrams remain a useful tool for mode identification in rotating stars but the tool must be used with care.
\end{abstract}

Key words. stars: $\beta$ Cephei variables - stars: oscillation - stars: rotation

\section{Introduction}

Mode identification, that is, determination of the radial order and spherical harmonic, is an essential step in asteroseismology. The task is not easy in the case of the oscillation frequency spectra in $\beta$ Cep and $\delta$ Sct stars, which are most often lacking equidistant patterns. The photometric diagnostic diagrams, i.e., the amplitude ratio vs. phase difference dependencies in different passbands, are the most popular tools for mode identification in pulsating stars. Following pioneering works (Balona \& Stobie 1979; Stamford \& Watson 1981) these tools have been applied mainly to $\beta$ Cep and $\delta$ Sct variables.

Theoretical diagnostic diagrams are based on linear nonadiabatic calculations of stellar oscillations and on models of static plane-parallel atmospheres. In the early works an arbitrary parametrization has been used instead of linear nonadiabatic calculations. This approach has been followed even in some recent studies (e.g. Garrido 2000). The nonadiabatic calculations were first included explicitly by Cugier et al. (1994) and subsequently by Balona \& Evers (1999), Cugier \& Daszyńska (2001), Balona et al. (2001) and also by Townsend (2002), who applied them to SPB stars.

Send offprint requests to: J. Daszyńska-Daszkiewicz, e-mail: daszynsk@camk.edu.pl
Up to now, the amplitudes and phases, which we - following Cugier et al. (1994) - call photometric nonadiabatic observables, were calculated in the framework of linear nonadiabatic theory, ignoring effects of rotation. However, amongst $\beta$ Cep and $\delta$ Sct stars slow rotators, for which such an approximation is adequate, are more an exception than a rule. Here we examine effects of moderate rotation on theoretical diagnostic diagrams. By moderate we mean so slow that perturbational treatment of rotation is adequate. Specifically, we rely on the third order formalism of Soufi et al. (1998).

The most important effect of rotation in the context of diagnostic diagrams is coupling between close frequency modes of spherical harmonic degree, $\ell$, differing by 2 . The effect was discussed in some detail by Soufi et al. (1998). The essential formulae are recalled in the next section of this paper.

Numerical results presented later concern one selected sequence of $\beta$ Cep models. On a qualitative level the results are applicable to all stars of this type. Our choice of $\beta$ Cep stars is motivated not only by the abundance of the observational data but also by the fact that we have credible results from linear nonadiabatic calculations. This is not true for $\delta$ Sct stars where there are serious uncertainties related to the treatment of convection.

Properties of unstable modes in the selected sequence of models are reviewed in Sect. 3. Also in this section we discuss 
the occurence of near resonances between two and three modes as well as certain consequences of mode coupling.

In Sect. 4 we discuss the visibility in various passbands of modes described by a single spherical harmonic over a wide range of $\ell$. Examples of diagnostic diagrams for coupled modes are given in Sect. 5 .

\section{Rotational mode coupling}

Chandrasekhar \& Lebovitz (1962) were the first to notice that rotation, even at its slow rate, significantly influences mode properties if there is a small frequency distance between modes with the same azimuthal order, $m$, and spherical harmonic degree, $\ell$, differing by 2 . In this case, each of the coupled modes must be represented by a certain superposition of spherical harmonics of the two modes involved. The effect was invoked to explain the nonradial character of pulsation in $\beta$ Cep stars. The effect of mode coupling has been subsequently discussed in a number of papers (e.g. Dziembowski \& Goode 1992; Soufi et al. 1998).

In the present paper we assume uniform rotation. Although our calculations were carried out to cubic order in the ratio of rotation to pulsation frequency, $\Omega / \omega$, for the purposes of this discussion and for the sake of simplicity, we will write formulae which are accurate only to $(\Omega / \omega)^{2}$. This is enough to capture the effects we want to review here.

In general, there may be more than two coupled modes. Then the displacement eigenfunction of an individual mode must be given as a sum

$\boldsymbol{\xi}=\sum_{k} a_{k} \boldsymbol{\xi}_{0 k}$

where $\boldsymbol{\xi}_{0 k}$ stands for the eigenfunctions in the spherical model. We choose the normalization in such a way that the radial component of the vector $\boldsymbol{\xi}_{0 k}$ is given by

$\xi_{0, r}=R Y_{l}^{m} \mathrm{e}^{-\mathrm{i} \omega t}$

The orthogonality condition is expressed in the form

$\int \mathrm{d}^{3} \boldsymbol{x}_{\rho} \boldsymbol{\xi}_{0 j} \cdot \boldsymbol{\xi}_{0 k}=\delta_{j k} I_{k}$

where $I_{k}$ is called mode inertia. The eigenfuncions and eigenvalues for coupled modes are calculated as a solution of the matrix equation,

$\left(\mathbf{B}-\omega^{2} \mathbf{E}\right) \boldsymbol{a}=\mathbf{0}$,

where $\mathbf{E}$ is a unit matrix and $\boldsymbol{a}=\left(a_{1}, a_{2}, \ldots\right)$ (see e.g. Soufi et al. 1998). The nondiagonal elements, $B_{j k}$, are of the order of $\Omega^{2}$ and are non-zero for the modes satisfying conditions $\ell_{j}=$ $\ell_{k} \pm 2$ and $m_{j}=m_{k}$. With our normalization these elements are not symmetric. The following relation is fulfilled:

$\frac{B_{k j}}{B_{j k}}=\frac{I_{j}}{I_{k}}$.

The diagonal elements are given by

$B_{k k}=\left(\omega_{0 k}+m C_{k} \Omega\right)^{2}+O\left(\Omega^{2}\right)$, where $\omega_{0 k}$ is the frequency in the spherically symmetric model and $C_{k}$ is the Ledoux constant. The $O\left(\Omega^{2}\right)$ term arises from quadratic effect of the Coriolis force and from the centrifugal distortion. The latter effect dominates for nonradial $p$-modes, as well as for $g$-modes of low orders.

Here we limit ourselves to couplings which involve up to three modes. The solution in the case of two-mode coupling is

$\omega_{ \pm}^{2}=\frac{B_{11}+B_{22} \pm \sqrt{\left(B_{11}-B_{22}\right)^{2}+4 B_{C}}}{2}$,

where $B_{\mathrm{C}}=B_{12} B_{21}$, and

$\left(\frac{a_{1}}{a_{2}}\right)_{ \pm}=\frac{B_{12}}{B_{11}-\omega_{ \pm}^{2}}$.

The coupling strength, which is measured by $B_{\mathrm{C}}$, depends on mode properties. Coupling between acoustic modes is stronger than that involving one or more gravity modes. This is so because the effect of the centrifugal distortion is only important in the acoustic cavity and it increases with the mode frequency.

For an exact resonance, that is when $B_{11}=B_{22}$, we get

$\left(\frac{a_{1}}{a_{2}}\right)_{ \pm}=\mp \operatorname{sign}\left(B_{12}\right) \sqrt{\frac{I_{2}}{I_{1}}}$,

and, regardless of the coupling strength, we have strong mode mixing. Then, the relative amplitudes are determined by mode inertiae. Therefore, for instance, an $\ell=2$ mode trapped in the interior thus having a large inertia may manifest itself as a radial mode. A close doublet of "radial" modes may be detected in this case.

Now we consider three-mode interaction, adopting $\ell_{2}=$ $\ell_{1}+2$ and $\ell_{3}=\ell_{2}+2$. An interesting situation arises when the closest resonance occurs between modes with $\ell$ differing by 4 . Since we have $B_{13}=B_{31}=0$, the interaction takes place through the $\ell_{2}$ mode. Let us consider a simple limiting case when $B_{11}=B_{33}$. Then, one solution is

$\omega^{2}=B_{11}=B_{33}, \quad a_{2}=0, \quad \frac{a_{1}}{a_{3}}=-\frac{B_{23}}{B_{21}}$.

The remaining two solutions are the same as in the case of two mode coupling, except that $B_{C}=B_{12} B_{21}+B_{23} B_{32}$. The highest degree mode, say $\ell=4$ or 5 , which is normally undetectable, acquires a large $\ell=0$ or, respectively, $\ell=1$ component. This means that some higher degree modes may become detectable by means of ground-based photometry.

\section{Close frequencies in a sequence of $\beta$ Cephei star models}

As an illustration we consider the evolutionary sequence of $12 M_{\odot}$ star models in the $\beta$ Cep instability strip. We adopted the standard chemical composition $(X=0.7, Z=0.02)$. Models were calculated not allowing for convective overshooting from the core. In these models we took into account the average effect of centrifugal force, assumed uniform rotation with an equatorial velocity of $100 \mathrm{~km} \mathrm{~s}^{-1}$ on the ZAMS, and imposed global angular momentum conservation during evolution. The adopted value of rotational velocity may be regarded typical for 

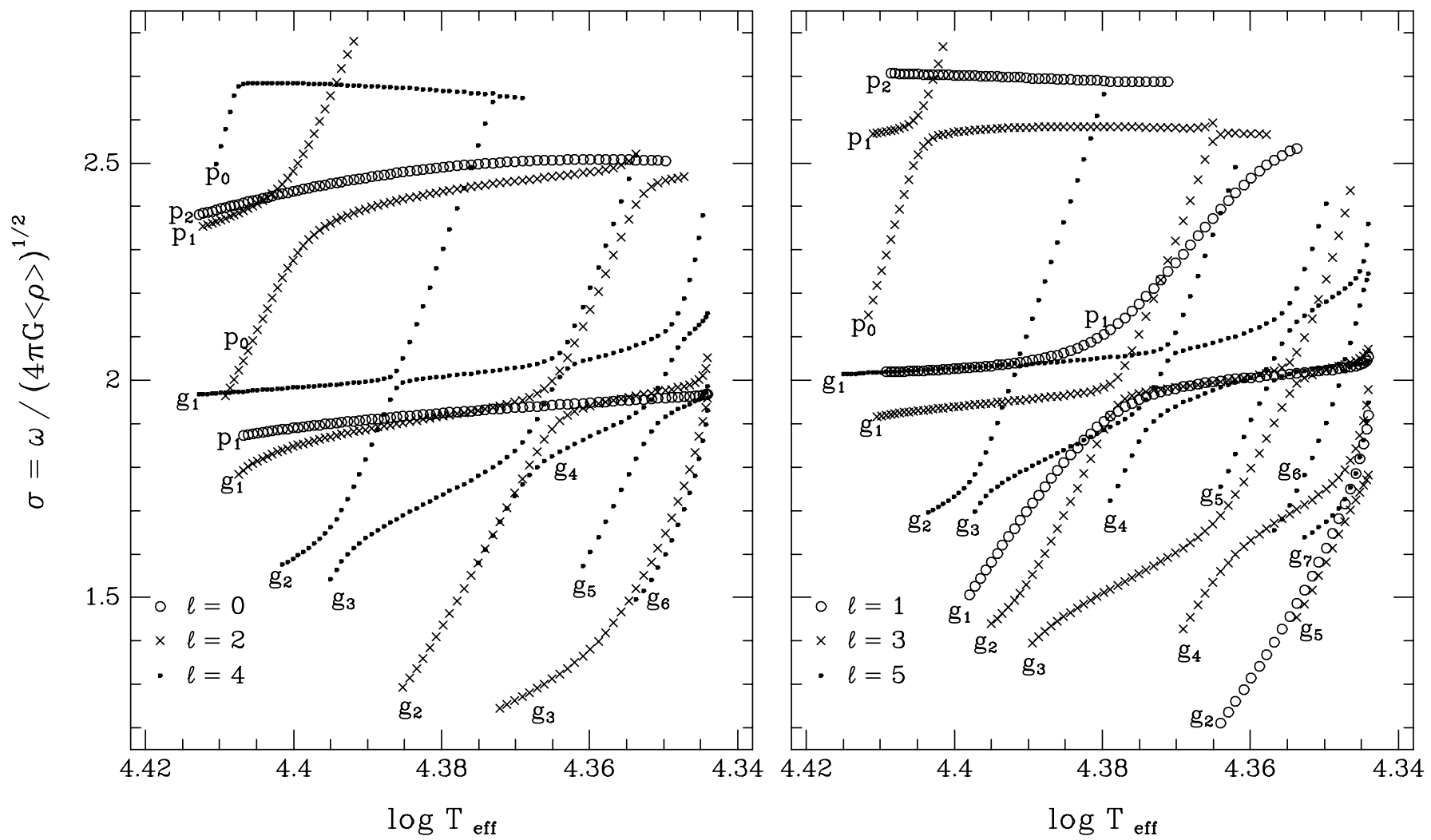

Fig. 1. Unstable low degree modes in a sequence of $\beta$ Cephei star models with mass of $12 M_{\odot}$. The models cover only the expansion phase of the main sequence evolution. In the left panel note that the $\ell=0, p_{1}$ and $\ell=2, g_{1}$ modes have close frequencies in the extended range of $\log T_{\text {eff }}$. At lower $\log T_{\text {eff }}$ the $\ell=2, g_{2}$ mode comes to a near resonance with the same radial mode. We see also the proximity of the nonradial $\ell=2$ and 4 modes at $\sigma \approx 1.6-1.8$. In the right panel, note a near resonance between the $\ell=1, g_{1}$ mode and, first, the $\ell=3, g_{2}$ and, later, the $g_{3}$. The proximity of three modes occurs only occasionally.

$\beta$ Cep stars. The coupling strength is proportional to the square of the velocity, but the result depends also on the frequency difference. Thus, we have no simple scaling of the effect of rotational mode coupling.

Figure 1 shows the frequency evolution in unstable lowdegree modes which are designated in accordance with the avoided-crossing principle. The physical nature of the modes, that is, the relative proportion of the contribution from acoustic and gravity propagation zones to mode's energy, is reflected in the slope of the $\sigma\left(\log T_{\text {eff }}\right)$ function. Slow and rapid rises correspond to dominant acoustic and gravity mode characters, respectively. The latter implies larger mode inertia.

We have to worry about mode coupling when the frequency distance between the modes becomes comparable to the rotational frequency. With our choice of rotation rate we have dimensionless rotational frequency $\sigma_{\text {rot }} \equiv \Omega / \sqrt{4 \pi G<\rho>} \approx 0.1$. Thus, we can see that in many instances the coupling may be significant.

Examples of coupled mode solutions are given in Table 1, where only zonal modes $(m=0)$ are included. The values of $\ell^{\prime}$ were assigned using again the avoided crossing principle, that is, the ordering of modes in frequencies was kept unchanged. The relevant frequency is $v_{\mathrm{NC}}$, which corresponds to $B_{k k}$. In most cases the value of $\ell^{\prime}$ identifies the dominant component in the surface amplitude. The exceptions occur for modes with drastically different inertiae.
The cases of exceptionally close two-mode resonances are 1, 3, 4, 7, 8, 9 and 11. There are differences between these cases, which reflect not only the differences in the proximity of the resonance but also in the nature of the coupled modes. As we have already discussed in Sect. 2, the coupling involving modes of gravity character is weaker than that involving only modes of the acoustic character. Weak coupling is reflected in the low values of $\Delta v_{\mathrm{C}}$ in cases 1 and 11 . However, the mixing of the modes is usually strong, except for $\ell^{\prime}=1$ mode in case 11 , which remains essentially pure $\ell=1$. This has to do with the disparity of inertiae for the coupled modes. In cases 3 and 4 both modes have $p$-mode character and the correction due to coupling is larger. Still, the frequency shift of $\sim 0.015 \mathrm{~d}^{-1}$ is significant only for data obtained with observations lasting longer than 2 months. In general, we find rather small rotation induced frequency changes, even in those cases when the coupling is large.

In some of the listed cases, like 2, 10 and 12, the coupling appears quite weak. Nonetheless, as we will see later in Sect. 5, the diagnostic diagrams may be significantly perturbed.

Among the three-mode couplings, case 9A most closely corresponds to the situation described in Sect. 2. With the accuracy adopted, the modes $\ell=1$ and 5 are in the exact resonance and the $\ell=3$ mode is not far away. Also in cases 3A and 7A, the three-mode coupling is important. In the latter case we see that $\ell^{\prime}=4$ mode acquires a large $\ell=0$ component. In case 
Table 1. Selected cases of rotational mode coupling. $\sigma_{0}$ is the dimensionless oscillation frequency in the spherical models, $v_{0}=2 \pi \omega_{0}$ is the corresponding cyclic frequency in $\mathrm{d}^{-1} ; v_{\mathrm{NC}}$ takes into account effects of rotation except for mode coupling; $\Delta v_{\mathrm{C}}$ is a frequency correction due to coupling, whereas $\Delta v_{\mathrm{T}}$ is a total frequency change relative to the spherical model; $a_{k}$ are amplitudes of the involved eigenmode components.

\begin{tabular}{|c|c|c|c|c|c|c|c|c|c|c|c|}
\hline Case & $\log T_{\text {eff }}$ & coupling & $\sigma_{0}$ & $v_{0}$ & $v_{\mathrm{NC}}$ & $\Delta v_{\mathrm{C}}$ & $\Delta v_{\mathrm{T}}$ & $\begin{array}{c}\text { coupled } \\
\text { modes }\end{array}$ & $a_{1}$ & $a_{2}$ & $a_{3}$ \\
\hline \multirow[t]{2}{*}{1} & 4.374 & $\ell_{1}=2, g_{2}$ & 1.611 & 3.836 & 3.849 & 0.002 & 0.014 & $\ell^{\prime}=2$ & 0.88 & -0.47 & \\
\hline & & $\ell_{2}=4, g_{4}$ & 1.610 & 3.834 & 3.846 & -0.002 & 0.011 & $\ell^{\prime}=4$ & 0.62 & 0.78 & \\
\hline \multirow[t]{2}{*}{2} & 4.406 & $\ell_{1}=0, p_{1}$ & 1.875 & 6.438 & 6.457 & 0.002 & 0.022 & $\ell^{\prime}=0$ & 1.00 & -0.07 & \\
\hline & & $\ell_{2}=2, g_{1}$ & 1.801 & 6.182 & 6.215 & -0.002 & 0.031 & $\ell^{\prime}=2$ & 0.14 & 0.99 & \\
\hline \multirow[t]{2}{*}{3} & 4.388 & $\ell_{1}=0, p_{1}$ & 1.913 & 5.265 & 5.282 & 0.013 & 0.030 & $\ell^{\prime}=0$ & 0.91 & -0.42 & \\
\hline & & $\ell_{2}=2, g_{1}$ & 1.894 & 5.214 & 5.248 & -0.013 & 0.021 & $\ell^{\prime}=2$ & 0.51 & 0.86 & \\
\hline \multirow[t]{3}{*}{$3 \mathrm{~A}$} & 4.388 & $\ell_{1}=0, p_{1}$ & 1.913 & 5.265 & 5.282 & 0.011 & 0.028 & $\ell^{\prime}=0$ & 0.92 & 0.36 & -0.15 \\
\hline & & $\ell_{2}=2, g_{1}$ & 1.894 & 5.214 & 5.248 & -0.014 & 0.020 & $\ell^{\prime}=2$ & 0.50 & -0.86 & 0.05 \\
\hline & & $\ell_{3}=4, g_{2}$ & 1.921 & 5.288 & 5.303 & 0.003 & 0.019 & $\ell^{\prime}=4$ & 0.59 & 0.51 & 0.63 \\
\hline \multirow[t]{2}{*}{4} & 4.374 & $\ell_{1}=0, p_{1}$ & 1.932 & 4.600 & 4.615 & -0.015 & 0.000 & $\ell^{\prime}=0$ & 0.85 & 0.53 & \\
\hline & & $\ell_{2}=2, g_{1}$ & 1.934 & 4.605 & 4.636 & 0.015 & 0.046 & $\ell^{\prime}=2$ & 0.58 & -0.82 & \\
\hline \multirow[t]{2}{*}{5} & 4.392 & $\ell_{1}=0, p_{2}$ & 2.462 & 7.096 & 7.110 & 0.008 & 0.022 & $\ell^{\prime}=0$ & 0.99 & -0.16 & \\
\hline & & $\ell_{2}=2, p_{0}$ & 2.385 & 6.877 & 6.913 & -0.008 & 0.028 & $\ell^{\prime}=2$ & 0.24 & 0.97 & \\
\hline \multirow[t]{2}{*}{6} & 4.376 & $\ell_{1}=0, p_{2}$ & 2.496 & 6.064 & 6.076 & 0.015 & 0.027 & $\ell^{\prime}=0$ & 0.95 & -0.30 & \\
\hline & & $\ell_{2}=2, p_{0}$ & 2.445 & 5.939 & 5.976 & -0.015 & 0.022 & $\ell^{\prime}=2$ & 0.39 & 0.92 & \\
\hline \multirow[t]{3}{*}{$6 \mathrm{~A}$} & 4.376 & $\ell_{1}=0, p_{2}$ & 2.496 & 6.064 & 6.076 & 0.015 & 0.027 & $\ell^{\prime}=0$ & 0.95 & 0.30 & 0.00 \\
\hline & & $\ell_{2}=2, p_{0}$ & 2.445 & 5.939 & 5.976 & -0.015 & 0.022 & $\ell^{\prime}=2$ & 0.39 & -0.92 & 0.00 \\
\hline & & $\ell_{3}=4, g_{1}$ & 2.491 & 6.050 & 6.058 & 0.000 & 0.009 & $\ell^{\prime}=4$ & 0.17 & -0.06 & -0.98 \\
\hline \multirow[t]{2}{*}{7} & 4.354 & $\ell_{1}=0, p_{2}$ & 2.508 & 4.875 & 4.886 & -0.019 & -0.009 & $\ell^{\prime}=0$ & 0.91 & 0.41 & \\
\hline & & $\ell_{2}=2, p_{0}$ & 2.521 & 4.899 & 4.925 & 0.019 & 0.046 & $\ell^{\prime}=2$ & 0.59 & -0.81 & \\
\hline \multirow[t]{3}{*}{$7 \mathrm{~A}$} & 4.354 & $\ell_{1}=0, p_{2}$ & 2.508 & 4.875 & 4.886 & -0.020 & -0.009 & $\ell^{\prime}=0$ & 0.91 & -0.42 & 0.00 \\
\hline & & $\ell_{2}=2, p_{0}$ & 2.521 & 4.899 & 4.925 & 0.020 & 0.046 & $\ell^{\prime}=2$ & 0.59 & 0.81 & 0.01 \\
\hline & & $\ell_{3}=4, g_{2}$ & 2.518 & 4.895 & 4.902 & -0.000 & 0.007 & $\ell^{\prime}=4$ & 0.71 & 0.27 & -0.64 \\
\hline \multirow[t]{2}{*}{8} & 4.370 & $\ell_{1}=1, g_{1}$ & 1.981 & 4.532 & 4.566 & -0.023 & 0.011 & $\ell^{\prime}=1$ & 0.73 & 0.68 & \\
\hline & & $\ell_{2}=3, g_{2}$ & 1.983 & 4.537 & 4.571 & 0.023 & 0.057 & $\ell^{\prime}=3$ & 0.67 & -0.75 & \\
\hline \multirow[t]{3}{*}{$8 \mathrm{~A}$} & 4.370 & $\ell_{1}=1, g_{1}$ & 1.981 & 4.532 & 4.566 & -0.018 & 0.016 & $\ell^{\prime}=1$ & 0.79 & -0.60 & -0.12 \\
\hline & & $\ell_{2}=3, g_{2}$ & 1.983 & 4.537 & 4.571 & 0.027 & 0.061 & $\ell^{\prime}=3$ & 0.61 & 0.79 & 0.10 \\
\hline & & $\ell_{3}=5, g_{4}$ & 1.940 & 4.439 & 4.456 & -0.009 & 0.009 & $\ell^{\prime}=5$ & 0.09 & -0.42 & 0.90 \\
\hline \multirow[t]{2}{*}{9} & 4.360 & $\ell_{1}=1, g_{1}$ & 2.006 & 4.144 & 4.180 & 0.022 & 0.058 & $\ell^{\prime}=1$ & 0.75 & -0.66 & \\
\hline & & $\ell_{2}=3, g_{2}$ & 2.008 & 4.148 & 4.178 & -0.022 & 0.007 & $\ell^{\prime}=3$ & 0.72 & 0.70 & \\
\hline \multirow[t]{3}{*}{$9 \mathrm{~A}$} & 4.360 & $\ell_{1}=1, g_{1}$ & 2.006 & 4.144 & 4.180 & 0.000 & 0.036 & $\ell^{\prime}=1$ & 0.87 & 0.00 & -0.49 \\
\hline & & $\ell_{2}=3, g_{2}$ & 2.008 & 4.148 & 4.178 & -0.044 & -0.014 & $\ell^{\prime}=3$ & 0.38 & -0.71 & 0.59 \\
\hline & & $\ell_{3}=5, g_{4}$ & 2.006 & 4.143 & 4.180 & 0.044 & 0.081 & $\ell^{\prime}=5$ & 0.39 & 0.69 & 0.61 \\
\hline \multirow[t]{2}{*}{10} & 4.406 & $\ell_{1}=1, p_{1}$ & 2.021 & 6.938 & 6.988 & 0.004 & 0.055 & $\ell^{\prime}=1$ & 1.00 & -0.08 & \\
\hline & & $\ell_{2}=3, g_{1}$ & 1.927 & 6.616 & 6.663 & -0.004 & 0.043 & $\ell^{\prime}=3$ & 0.15 & 0.99 & \\
\hline \multirow[t]{3}{*}{$10 \mathrm{~A}$} & 4.406 & $\ell_{1}=1, p_{1}$ & 2.021 & 6.938 & 6.988 & 0.002 & 0.053 & $\ell^{\prime}=1$ & 0.97 & 0.05 & -0.25 \\
\hline & & $\ell_{2}=3, g_{1}$ & 1.927 & 6.616 & 6.663 & -0.013 & 0.034 & $\ell^{\prime}=3$ & 0.14 & -0.96 & 0.23 \\
\hline & & $\ell_{3}=5, g_{1}$ & 2.022 & 6.940 & 7.007 & 0.011 & 0.077 & $\ell^{\prime}=5$ & 0.21 & 0.12 & 0.97 \\
\hline \multirow[t]{2}{*}{11} & 4.372 & $\ell_{1}=1, p_{1}$ & 2.230 & 5.205 & 5.224 & 0.000 & 0.019 & $\ell^{\prime}=1$ & 1.00 & 0.05 & \\
\hline & & $\ell_{2}=3, g_{1}$ & 2.231 & 5.208 & 5.218 & -0.000 & 0.009 & $\ell^{\prime}=3$ & 0.66 & -0.75 & \\
\hline \multirow[t]{2}{*}{12} & 4.380 & $\ell_{1}=1, p_{2}$ & 2.690 & 6.795 & 6.858 & 0.009 & 0.072 & $\ell^{\prime}=1$ & 0.99 & -0.13 & \\
\hline & & $\ell_{2}=3, p_{0}$ & 2.584 & 6.528 & 6.572 & -0.009 & 0.035 & $\ell^{\prime}=3$ & 0.21 & 0.98 & \\
\hline \multirow[t]{3}{*}{$12 \mathrm{~A}$} & 4.380 & $\ell_{1}=1, p_{2}$ & 2.690 & 6.795 & 6.858 & 0.009 & 0.072 & $\ell^{\prime}=1$ & 0.99 & 0.13 & 0.00 \\
\hline & & $\ell_{2}=3, p_{0}$ & 2.584 & 6.528 & 6.572 & -0.009 & 0.035 & $\ell^{\prime}=3$ & 0.21 & -0.98 & 0.00 \\
\hline & & $\ell_{3}=5, g_{2}$ & 2.659 & 6.717 & 6.726 & 0.000 & 0.008 & $\ell^{\prime}=5$ & 0.08 & -0.17 & -0.98 \\
\hline
\end{tabular}


10A the frequency distance between $\ell=1$ and 5 is small, but because the distance to $\ell=3$ is relatively large, the coupling is essentially inconsequential. Also in case $6 \mathrm{~A}$, the coupling to the third mode is without significant consequences. Here the reason is the gravity character of $\ell=4$ mode.

Coming to tesseral modes $(m \neq 0)$, we first note that the coupling occurs if $m= \pm 1$ and, for even $\ell$ modes, also if $m= \pm 2$. In general, coupling conditions between prograde and retrograde mode pairs are not the same. The asymmetry is due to the difference in the Ledoux constant for the coupled modes. The only systematic difference relative to the $m=0$ modes is a somewhat weaker coupling due to smaller value of the angular integrals entering expressions for $B_{j k}$.

The near degeneracy of rotationally coupled modes occurs also in wide ranges of $\delta$ Sct star models. For high order $p$ modes the effect is systematic, as implied by the asymptotic relation $\omega_{n, \ell+2} \approx \omega_{n-1, \ell}$. Since the coupling strength increases with frequency, in the high order $p$-mode pulsators the effect begins to be significant at lower rotation rates than in $\beta$ Cep stars. Frequency changes caused by rotational mode coupling in $\delta$ Sct stars and in solar type pulsators have been discussed by Goupil et al. (2000) and by Dziembowski \& Goupil (1998), respectively.

\section{Photometric diagnostic diagrams for single spherical harmonic modes}

Our approach follows that of Cugier et al. (1994), except for correcting an error in the expression for perturbed gravity, which fortunately has negligible consequences (Cugier \& Daszyńska 2001). Here we outline the main steps. In our construction of theoretical diagnostic diagrams we rely on the linear nonadiabatic description of oscillations. Normalization of eigenfunctions is fixed in Eq. (2). With that, the local displacement of the photosphere in the stellar reference frame may be written as follows

$\delta r(R, \theta, \varphi)=\varepsilon R \operatorname{Re}\left\{Y_{\ell}^{m} \mathrm{e}^{-\mathrm{i} \omega t}\right\}$,

where $\varepsilon$ is a small quantity, which is determined by data. This equation implies that the phase zero corresponds to the maximum of displacement. The use of $-\mathrm{i} \omega t$ in the exponential time dependence, which is now the most common choice, instead of $+\mathrm{i} \omega t$ used by Cugier et al. (1994), results in the opposite signs for the phase differences between calculated observables. For a comparison of calculated and measured values it is important to check the sign convention adopted in the data analysis. With the sign convention adopted in the present work, the phase difference $\phi_{a}-\phi_{b}>0$ means that the maximum of $a$ occurs after the maximum of $b$.

The corresponding changes of the effective temperature and gravity during the pulsation cycle are given by

$\frac{\delta T_{\mathrm{eff}}}{T_{\mathrm{eff}}^{0}}=\varepsilon \frac{1}{4} \operatorname{Re}\left\{f Y_{\ell}^{m} \mathrm{e}^{-\mathrm{i} \omega t}\right\}$,

and

$\frac{\delta g_{\mathrm{eff}}}{g_{\mathrm{eff}}^{0}}=-\left(2+\frac{3 \omega^{2}}{4 \pi G<\rho>}\right) \frac{\delta r}{R}$,

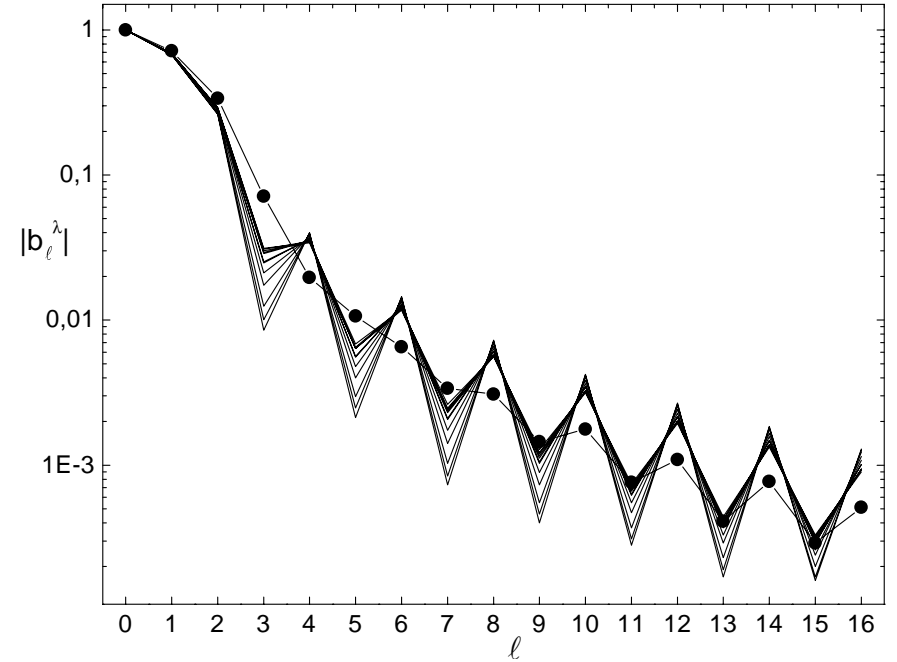

Fig. 2. The absolute values of the disc averaging factor plotted for bolometric and for twelve passbands as a function of $\ell$. The passbands include $u, v, b, y$ and $U, B, V, R, I, J, H, K$ filters.

respectively, where $f$ is the complex quantity determined from linear nonadiabatic calculations, $\langle\rho\rangle$ is the mean density of the star and $G$ is the gravitational constant.

Further, we assume static plane-parallel atmosphere, and we write the monochromatic flux variation caused by the oscillation mode with $\ell$ degree in the following form:

$$
\begin{gathered}
\frac{\Delta \mathcal{F}_{\lambda}}{\mathcal{F}_{\lambda}^{0}}=\varepsilon Y_{\ell}^{m}(i, 0) b_{\ell}^{\lambda} \operatorname{Re}\left\{\left[D_{1, \ell}^{\lambda}+D_{2, \ell}+D_{3, \ell}^{\lambda}\right] \mathrm{e}^{-\mathrm{i} \omega t}\right\}, \\
D_{1, \ell}^{\lambda}=\frac{1}{4} f \frac{\partial \log \left(\mathcal{F}_{\lambda}\left|b_{\ell}^{\lambda}\right|\right)}{\partial \log T_{\mathrm{eff}}}, \\
D_{2, \ell}=(2+\ell)(1-\ell), \\
D_{3, \ell}^{\lambda}=-\left(\frac{3 \omega^{2}}{4 \pi G<\rho>}+2\right) \frac{\partial \log \left(\mathcal{F}_{\lambda}\left|b_{\ell}^{\lambda}\right|\right)}{\partial \log g_{\mathrm{eff}}^{0}}
\end{gathered}
$$

and

$b_{\ell}^{\lambda}=\int_{0}^{1} h_{\lambda}^{0}(\mu) \mu P_{\ell}(\mu) \mathrm{d} \mu$.

In this formula, the $D_{1, \ell}^{\lambda}$ term describes the temperature effects, whereas the influence of the gravity changes is contained in the $D_{3, \ell}^{\lambda}$ term. Both include the perturbation of the limb-darkening. In our calculation we relied on Claret's (2000) analytical fit to Kurucz's (1998) tabular data. The $\ell$-dependence of $D_{1}$ and $D_{3}$ arises from the nonlinearity of the adopted limb-darkening law. The $D_{2, \ell}$ term stands for the geometrical effects. The effect of orientation with respect to the observer is described by the spherical harmonic $Y_{\ell}^{m}(i, 0)$.

In Fig. 2 we show the dependence of the disc averaging factor, $b_{\ell}^{\lambda}$, on mode degree, $\ell$, for different passbands. For comparison, the bolometric values are also shown. These are similar to those calculated by Dziembowski (1977) with the Eddington's limb darkening law. In contrast, large differences are seen for individual passbands. Stronger averaging of odd $-\ell$ modes gives rise to a pronounced oscillatory dependence on $\ell$, which is superimposed on the decreasing trend. The amplitude is larger 


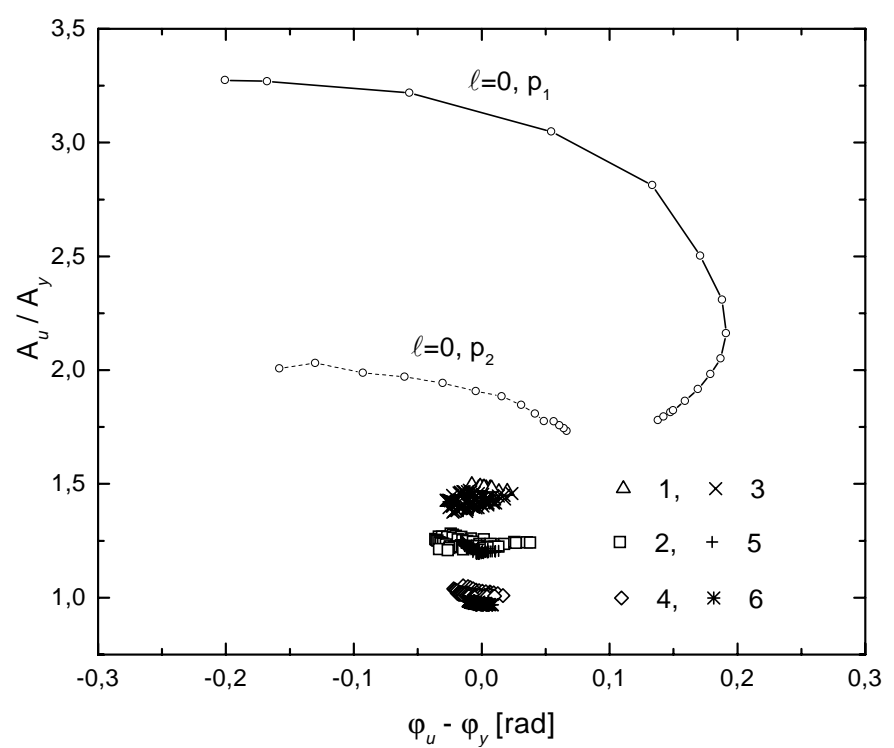

Fig. 3. Theoretical diagnostic diagram with the Strömgren passbands $u$ and $y$ for unstable low degree modes in the $\beta$ Cep star models with $M=12 M_{\odot}$. In the sequences for the $\ell=0$ modes the most negative values of the phase difference correspond to the coolest models.

than for the bolometric flux due to the flatter limb-darkening law. Note that in the case of uniform brightness disc, the odd- $\ell$ modes beginning with $\ell=3$ are completely averaged out.

The decreasing trend does not properly reflect the $\ell$ dependence of the expected amplitudes. This would be true only if the temperature term dominates. For modes of low radial orders the geometrical term $D_{2}$ becomes dominant around $\ell=6$. Then owing to the $(\ell+2)(\ell-1)$ factor the decline with $\ell$ is slower than implied by Fig. 2 .

For calculating diagnostic diagrams it is convenient to use the amplitude of the monochromatic flux variation in the following complex form

$A_{\lambda}(i)=\varepsilon Y_{\ell}^{m}(i, 0) b_{\ell}^{\lambda}\left(D_{1, \ell}^{\lambda}+D_{2, \ell}+D_{3, \ell}^{\lambda}\right)$

which is equivalent to Eq. (14). From this expression we can directly obtain the amplitude ratio and phase difference in any selected pair of passbands. Our choice is $u$ and $y$ Strömgren filters. For modes described by a single $Y_{\ell}^{m}$, these photometric observables are independent of the aspect angle and azimuthal order, $m$.

The diagnostic diagram for unstable modes in our model sequence is presented in Fig. 3. Modes up to $\ell=6$ are included. We can see that, in contrast to the radial modes, the nonradial modes are crowded in small areas. Two aspects of this plot call for an explanation. Firstly, why it is that the amplitude ratios and phase differences of radial modes are particularly dependent on frequency and model temperature? Secondly, what is the origin of the grouping of nonradial modes?

The main reason for this distinctive behavior of radial modes is a comparable but opposite contribution of the temperature and the geometrical terms to luminosity variation. The sum of the real part of $D_{1}^{\lambda}$ and $D_{2}$ varies significantly with temperature and it is strongly frequency dependent. For $\ell=1$, we have $D_{2}=0$ and the only reason for the spread of the mode positions is a weak dependence of $b_{\ell}^{\lambda}$ on the passbands. The role of the geometrical term for $\ell=2$ modes is comparable to that of $D_{1}^{\lambda}$. However, the two terms add, which results in much smaller spread of their positions. The near overlap of $\ell=1$ and 3 modes is coincidencial. At still higher $\ell$, when geometrical effect dominates, the even and odd degree modes are gathered in two small separate domains, determined solely by the $b_{\ell}^{\lambda}$ factor (see Fig. 2). The phase difference is always close to zero owing to the small role of the only complex term, $D_{1}^{\lambda}$.

\section{Photometric diagrams for coupled modes}

The complex amplitude of the monochromatic flux variation for a coupled mode may be expressed in the form

$\mathcal{A}_{\lambda}(i)=\sum_{k} a_{k} A_{\lambda, k}(i)$

where $a_{k}$ are solutions of Eq. (4) and $A_{\lambda}(i)$ is given in Eq. (17). The quantities $a_{k}$ describe contributions of the $\ell_{k}$-modes to the coupled mode $\ell^{\prime}$. Selected values of $a_{k}$ are given in Table 1. The quantity $f$ appearing in Eq. (17) is only weakly dependent on $\ell$. Its relatively strong dependence on frequency is irrelevant in view of very small frequency spread of the mode involved. In contrast to the amplitude ratio and phase difference for the pure modes, these quantities now depend on the inclination angle, $i$, and the azimuthal order, $m$. We will show here few representative examples of the photometric diagnostic diagrams in the case of two and three mode coupling and we will discuss the consequence for the mode identification.

Naively, one could expect that the coupled mode appears in the diagnostic diagrams between positions corresponding to its components associated with individual spherical harmonics. We will see that this is not true.

As our first illustrative example, we chose the case when the $\ell=0$ and 2 modes are in close resonance (case 4 in Table 1). In Fig. 4 we show how positions of the coupled modes move in the diagnostic diagram with varying aspects. A comparison of plots in the upper and lower panels reveals that the movement is strongly mode-dependent. Let us focus first on the case of mode dominated by the radial component, shown in the upper panel. Due to close resonance, contamination with the $\ell=2$ component is significant. The amplitudes of the $\ell=0$ and 2 components are 0.85 and 0.53 , respectively. An observer from the polar direction will identify this mode as $\ell=1$. This is so, because the main role of the $\ell=2$ component is a cancellation of the geometrical term in the expression for the flux variation (see Eq. (14)). When the observer moves away toward the equatorial direction, he will see the mode as a pure $\ell=0$, at the angle corresponding to the node of $Y_{2}^{0}(i, 0)$, and subsequently he will get mode characteristics which do not correspond to any of single- $\ell$ modes. Note that such an appearance has a significant probability, as measured by the density of dots.

Figure 5 helps us to understand the cause of the described behaviour. Here we show complex, arbitrarily normalized, amplitudes in the two selected passbands. To make the amplitudes realistic one should divide them by a factor between 10 to 100 . We see an increasing phase difference between two passbands 

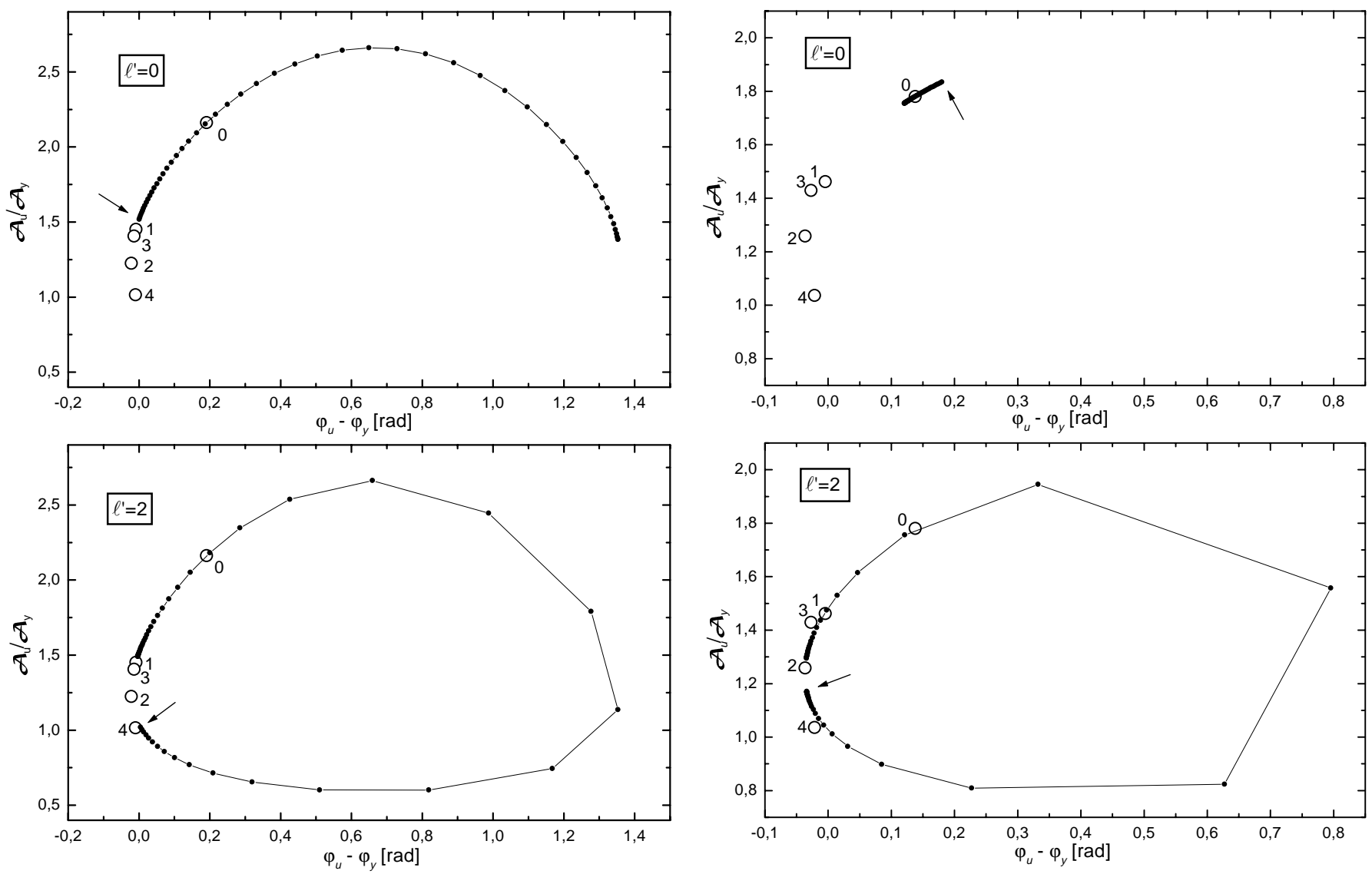

Fig. 4. The diagnostic diagrams with Strömgren photometry passbands $u$ and $y$, showing positions for coupled $\ell=0, p_{1}$ and $\ell=2, g_{1}$ modes (case 4 in Table 1) in the model at $\log T_{\text {eff }}=4.3741$ (see Fig. 1). This model corresponds to the smallest frequency distance between these two modes. The upper panel refers to the solution dominated by the $\ell=0$ component, while the lower one refers to the solution dominated by $\ell=2$ component. Arrows correspond to observations from the polar direction. Spacing between consecutive dots is 0.02 in $\cos i$. Note that the density of dots reflects probability of observing the modes in various parts of that diagram. Circles indicate positions of single $\ell$ modes with $\ell=0,1,2,3,4$.

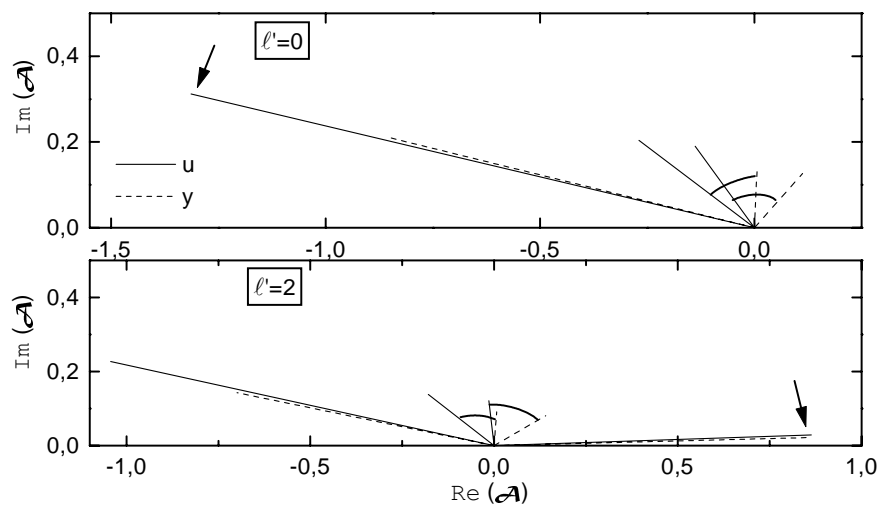

Fig. 5. Complex amplitudes in Strömgren passbands $u, y$ for modes used in Fig. 4. The three pairs of vectors shown in the upper panel correspond to $\cos i=1.0,0.32$ and 0.0 . The four pairs of vectors in the lower panel correspond to $\cos i=0.0,0.66,0.72$ and 1.0. The arrows mark amplitudes seen from the polar direction.

Fig. 6. The same as in Fig. 4 but the coupling between modes $\ell=0$ and 2 is much weaker (case 2 in Table 1).

when we move from polar to equatorial direction. This is predominantly due to a decrease of the real part of the complex amplitude in $y$ passband. Why does the argument for the $y$ passband change much more rapidly with aspect than that for $u$ passband? This is due to a smaller absolute value of $D_{1}$, hence the greater role of $D_{2}$. Only in this colour does $\operatorname{Re}(\mathcal{A})$ pass through zero. This happens at $\cos i=0.32$. Note, however, that when the phase difference is large, the pulsation amplitude is rather small. So that the situation may not be easily observable.

The very different pattern of the $\ell^{\prime}=2$ mode behaviour, shown in lower panel of Fig. 4, is due to the fact that now the real part of the amplitude in both colours changes sign. This is caused by the greater contribution from the $\ell=2$ component. Here the equatorial position of the mode is not so distant from the polar one. Surprisingly, the mode is often seen as either $\ell=1$ or $\ell>3$.

Figure 6 illustrates case 2, when as we have noted the coupling is rather weak. Whereas for the $\ell^{\prime}=0$ root the effect of the coupling is marginal, for $\ell^{\prime}=2$ it is quite significant. In spite of the fact that the mode is dominated by $\ell=2$ component, it has a fairly high chance to be identified as $\ell=1$ or $\ell>3$.

The cases of $\ell=1$ and 3 coupling never lead to a large departure of the $\ell^{\prime}=1$ modes from the $\ell=1$ position. Since mode $\ell^{\prime}=3$ acquires a substantial $\ell=1$ component, it is mostly seen through this component and hence would be 

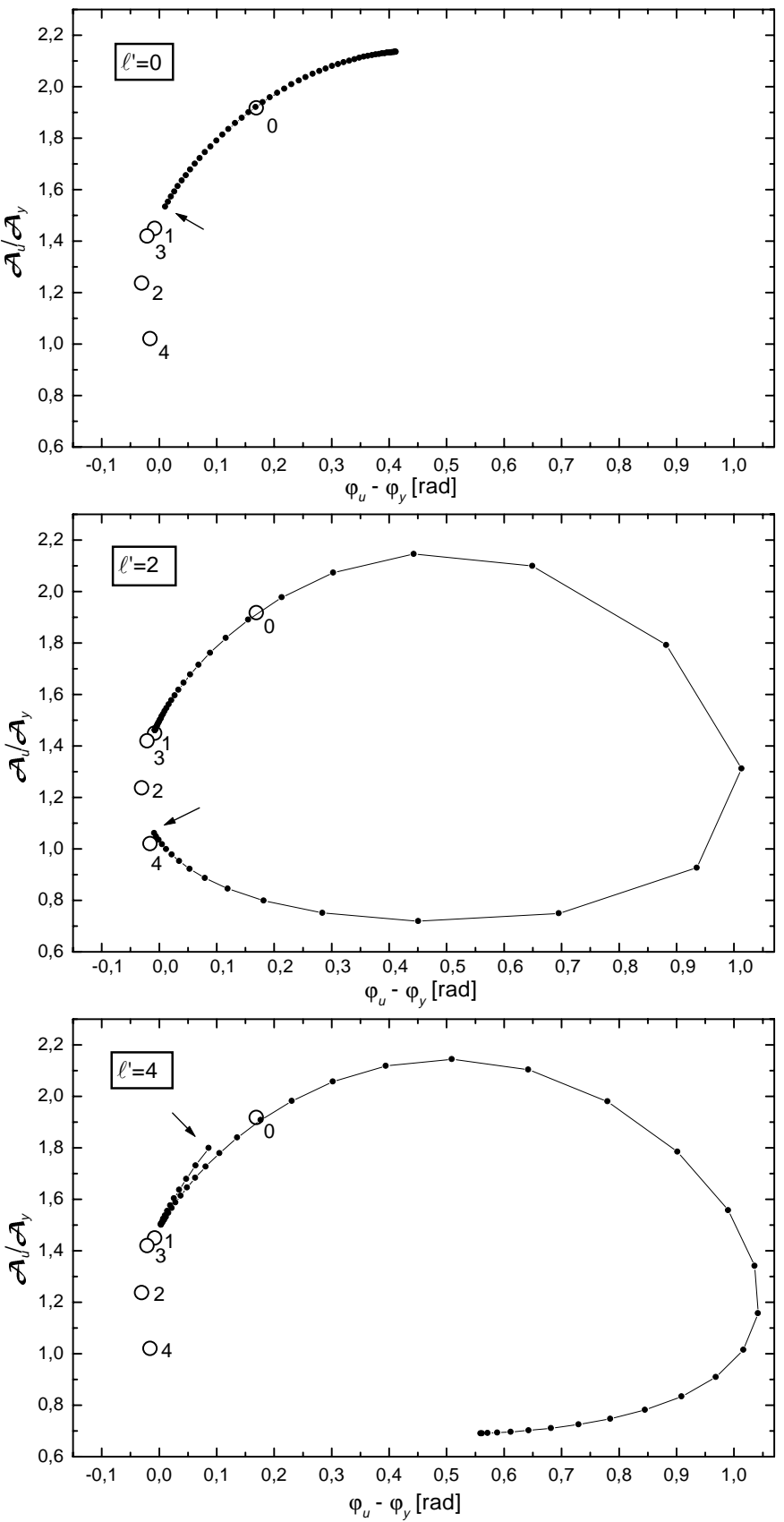

Fig. 7. An example of the diagnostic diagrams for the three mode coupling (case 3A).

identified as $\ell=1$. It is interesting that many different modes in rotating stars may be identified as $\ell=1$ by means of the diagnostic diagram method, if effects of rotation are ignored.

Figure 7 illustrates an interesting case (case $3 \mathrm{~A}$ in Table 1) of the three-mode coupling. The $\ell^{\prime}=0$ mode is most often seen close to the $\ell=0$ position, but there is also a fair probability to see this mode close the $\ell=1$ position. The path for the $\ell^{\prime}=2$ mode is very similar to that shown in the lower panel of Fig. 5. The $\ell^{\prime}=4$ mode is most often seen between $\ell=0$ and $\ell=1$ positions. However, seen from the equatorial direction it is quite far from any position of a single $\ell$ mode.
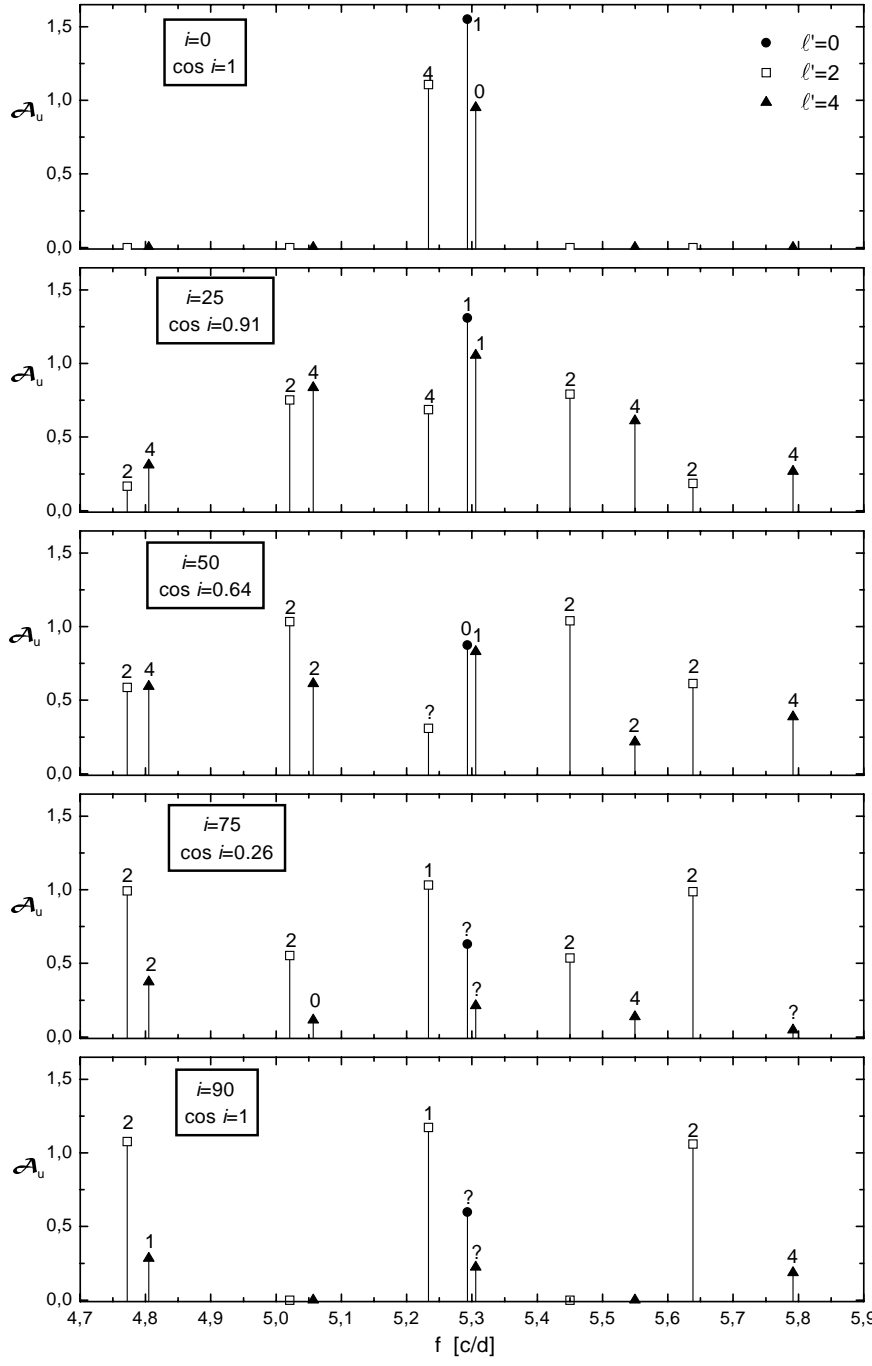

Fig. 8. Multiplets of coupled $\ell^{\prime}=0,2,4$ modes for case $3 \mathrm{~A}$. Amplitudes in the $u$ band were calculated assuming common normalization, $\varepsilon=1$ in Eq. (17). Numbers above the symbols give most likely identification upon neglecting effects of rotation. Lack of numbers means that mode can not be identified with any $\ell$.

For other modes of the same multiplets the coupling effects are less spectacular. In all the cases from $m=-2$ to $m=+2$, the $\ell^{\prime}=2$ modes hardly move from the $\ell=2$ position. As expected, the effect for the $\ell^{\prime}=4$ mode is more significant. The mode stays predominantly between the $\ell=1$ and $\ell=4$ positions.

Figure 8 shows how mode-coupling of the multiplets would be identified if rotation is neglected. In this figure we plotted the observed amplitudes assuming that the intrinsic mode amplitudes are the same. Note that the indicated amplitudes should be divided by a factor between 10 and 100 to correspond to reality. Identifications were based on the diagram in Fig. 3.

\section{Conclusions}

We have seen that even at moderate rotation, mode coupling leads to complications in the diagnostic diagrams used for 
photometric mode identification. Modes of degrees $\ell>2$, which are often ignored in identification of peaks in oscillation spectra, may acquire substantial low $\ell$ components, and are more easily detected. Unlike modes described by a single spherical harmonic, the positions of coupled modes in the diagnostic diagrams depend on the aspect and on the azimuthal order. The positions may be quite confusing, for instance, a mode composed of the $\ell=0$ and $\ell=2$ components may appear for a range of aspect angles at the $\ell=1$ position.

All this is not good news for the photometric mode identification procedure. The problems are not confined to a few cases, but occur at the typical rotation rates encountered in $\beta$ Cep and $\delta$ Sct stars. Close frequencies of rotationally coupled modes occur over wide ranges of the instability strips. The implication is that we must be careful in using the diagnostic diagrams for inferring the $\ell$ values. The diagrams remain useful. After all, they do provide observational constrains on stars and their oscillations. However, mode identification may be done only simultaneously with determination of stellar parameters and inclination of rotational axis.

Acknowledgements. J.D.-D. gratefully acknowledges hospitality of the Copernicus Astronomical Center staff during her postdoctoral stay. The work was supported by KBN grant No. 5 P03D 01220.

\section{References}

Balona, L. A., \& Stobie, R. S. 1979, MNRAS, 189, 649

Balona, L. A., \& Evers, E. A. 1999, MNRAS, 302, 349

Balona, L. A., Bartlett, B., Caldwell, J. A. R., et al. 2001, MNRAS, 321,239

Claret, A. 2000, A\&A, 363, 1081

Chandrasekhar, S., \& Lebovitz, N. R. 1962, ApJ, 136, 1105

Cugier, H., \& Daszyńska, J. 2001, A\&A, 377, 113

Cugier, H., Dziembowski, W. A., \& Pamyatnykh, A. A. 1994, A\&A, 291,143

Dziembowski, W. A. 1977, Acta Astron., 27, 203

Dziembowski, W. A., \& Goode, P. R. 1992, ApJ, 394, 670

Dziembowski, W. A., \& Goupil, M.-J. 1998, in Workshop on Science with Small Space Telescopes, ed. T. R. Bedding, \& H. Kjeldsen (University of Aarhus), 69

Garrido, R. 2000, in Delta Scuti and Related Stars, Proc. of the 6th Vienna Workshop in Astrophysics, ed. M. Breger, \& M. H. Montgomery, ASP Conf. Ser., 210, 67

Goupil, M.-J., Dziembowski, W. A., Pamyatnykh, A. A., \& Talon, S. 2000, in Delta Scuti and Related Stars, Proc. of the 6th Vienna Workshop in Astrophysics, ed. M. Breger, \& M. H. Montgomery, ASP Conf. Ser., 210, 267

Kurucz, R. L. 1998, CD-ROM, Nos. 13 and 19

Soufi, F., Goupil, M.-J., \& Dziembowski, W. A. 1998, A\&A, 334, 911

Townsend, R. D. 2002, MNRAS, 330, 855

Watson, R. D. 1988, Ap\&SS, 140, 255 